\title{
Whole-genome re-sequencing for the identification of high contribution susceptibility gene variants in patients with type 2 diabetes
}

\author{
XIAOJUAN SUN ${ }^{1,2^{*}}$, WEIGUO SUI ${ }^{3 *}$, XIAOBING WANG ${ }^{2}$, XIANLIANG HOU ${ }^{3}$, \\ MINGLIN OU ${ }^{3}$, YONG DAI ${ }^{4}$ and YUEYING XIANG ${ }^{2}$
}

\begin{abstract}
${ }^{1}$ Department of Social Medicine and Health Service Management, College of Military Preventive Medicine, Third Military Medical University, Chongqing 400038; ${ }^{2}$ Department of Health Management Center, 181st Hospital; ${ }^{3}$ Nephrology Department, 181st Hospital and Guangxi Key Laboratory of Metabolic Diseases Research, Guilin, Guangxi 541002;

${ }^{4}$ Department of Clinical Medical Research Center, The Second Clinical Medical College of Jinan University, Shenzhen People's Hospital, Shenzhen, Guangdong 518020, P.R. China
\end{abstract}

Received March 17, 2015; Accepted January 21, 2016

DOI: $10.3892 / \mathrm{mmr} .2016 .5014$

\begin{abstract}
There is increasing evidence that several genes are associated with an increased risk of type 2 diabetes (T2D); genome-wide association investigations and whole-genome re-sequencing investigations offer a useful approach for the identification of genes involved in common human diseases. To further investigate which polymorphisms confer susceptibility to T2D, the present study screened for high-contribution susceptibility gene variants Chinese patients with T2D using whole-genome re-sequencing with DNA pooling. In total, 100 Chinese individuals with T2D and 100 healthy Chinese individuals were analyzed using whole-genome re-sequencing using DNA pooling. To minimize the likelihood of systematic bias in sampling, paired-end libraries with an insert size of $500 \mathrm{bp}$ were prepared for in T2D in all samples, which were then subjected to whole-genome sequencing. Each library contained four lanes. The average sequencing depth was 35.70. In the present study, $1.36 \mathrm{~GB}$ of clean sequence data were generated, and the resulting calculated T2D genome consensus sequence covered $99.88 \%$ of the hg19 sequence. A total of
\end{abstract}

Correspondence to: Mr. Yong Dai, Clinical Medical Research Center, The Second Clinical Medical College of Jinan University, Shenzhen People's Hospital, 1017 Dongmen North Road, Shenzhen, Guangdong 518020, P.R. China

E-mail: daiyong2222@gmail.com; daiyong22@aliyun.com

Mr. Yueying Xiang, Department of Health Management Center, 181st Hospital, 1 Xinqiaoyuan Road, Guilin, Guangxi 541002, P.R. China

E-mail: xiangyy181@163.com

*Contributed equally

Key words: whole-genome re-sequencing, type 2 diabetes, DNA pooling, bioinformatics, functional genomics
3,974,307 single nucleotide polymorphisms were identified, of which $99.88 \%$ were in the dbSNP database. The present study also found 642,189 insertions and deletions, 5,590 structure variants (SVs), 4,713 copy number variants (CNVs) and 13,049 single nucleotide variants. A total of 1,884 somatic CNVs and 74 somatic SVs were significantly different between the cases and controls. Therefore, the present study provided validation of whole-genome re-sequencing using the DNA pooling approach. It also generated a whole-genome re-sequencing genotype database for future investigations of T2D.

\section{Introduction}

Type 2 diabetes (T2D) is a complex, multifactorial disorder characterized by chronic hyperglycemia due to the interplay of multiple genetic variants and several environmental factors. As a result of aging populations, and the increasing prevalence of obesity and physical inactivity, the number of patients with T2D has markedly increased worldwide (1). The disease is considered a polygenic disorder, in which each genetic variant confers a partial and additive effect. Only $5-10 \%$ of T2D cases are due to single gene defects; these include maturity-onset diabetes of the young, insulin resistance syndromes, mitochondrial diabetes and neonatal diabetes (2). Examining T2D susceptibility genes may be useful for the prediction, prevention and early treatment of the disease.

Following previous genome-wide association studies (GWAS), the number of replicated common genetic variants associated with T2D has rapidly increased (3-9). In addition, $>40$ T2D-associated genetic loci have been identified, however, these loci have been revealed primarily on the basis of investigations of European individuals (10). The identified genomes only explain a small proportion of the estimated heritability of T2D, suggesting that additional genetic factors remain to be identified. One limitation of GWAS is the large number of hypotheses and the high economic cost of these 
Table I. Clinical and biochemical characteristics of the 200 individuals recruited for re-sequencing.

\begin{tabular}{lrr}
\hline Characteristic & ND $(\mathrm{n}=100)$ & T2D $(\mathrm{n}=100)$ \\
\hline Gender (males/females) & $63 / 37$ & $55 / 45$ \\
Age (years) & $60.1 \pm 11.8$ & $5.4 \pm 0.6$ \\
HbA1c (\%) & $5.4 \pm 0.6$ & $9.6 \pm 2.3$ \\
Fasting plasma glucose (mmol/l) & $4.9 \pm 0.6$ & $11.4 \pm 3.9$ \\
2-h OGTT-based plasma glucose (mmol/l) & $6.0 \pm 0.4$ & $13.5 \pm 2.1$ \\
Body mass index (kg/m $\left.{ }^{2}\right)$ & $24.0 \pm 1.5$ & $31.7 \pm 5.0$ \\
Waist circumference (cm) & $82.3 \pm 6.7$ & $105.0 \pm 9.8$ \\
Systolic blood pressure $(\mathrm{mmHg})$ & $125.0 \pm 7.0$ & $152.0 \pm 9.0$ \\
Diastolic blood pressure $(\mathrm{mmHg})$ & $84.0 \pm 5.0$ & $92.0 \pm 6.0$ \\
\end{tabular}

Data are presented as the mean \pm standard deviation for normally distributed traits, or the median. ND, non-diabetic; T2D, type 2 diabetes; OGTT, oral glucose tolerance test.

Table II. Quality control of sequencing data.

\begin{tabular}{|c|c|c|c|}
\hline \multirow[b]{2}{*}{ Category } & \multicolumn{2}{|c|}{ Data } & \multirow[b]{2}{*}{ Discarded reads $(\mathrm{n})$} \\
\hline & Raw & Clean & \\
\hline Reads (n) & $1,442,754,024$ & $1,367,776,414$ & \\
\hline Data size (bp) & $129,847,862,160$ & $123,099,877,260$ & \\
\hline $\mathrm{N}$ of $\mathrm{fq} 1(\mathrm{n})$ & $41,142,158$ & $1,257,883$ & \\
\hline $\mathrm{N}$ of fq2 (n) & $130,903,222$ & $3,072,470$ & \\
\hline GC of fq1 $(\%)$ & $39.62-39.82$ & $39.47-39.7$ & \\
\hline $\mathrm{GC}$ of fq2 $(\%)$ & 39.69-39.97 & $39.56-39.78$ & \\
\hline Q20 of fq1 (\%) & $96.16-97.06$ & $97.12-97.76$ & \\
\hline Q20 of fq2 (\%) & $90.02-93.33$ & $93.88-95.97$ & \\
\hline Q30 of fq1 (\%) & $90.13-92.30$ & $91.28-93.20$ & \\
\hline $\mathrm{Q} 30$ of $\mathrm{fq} 2(\%)$ & $82.31-87.61$ & $86.04-90.21$ & \\
\hline Discarded reads associated with $\mathrm{N}$ & & & $4,639,892$ \\
\hline Discarded reads due to low quality bases & & & $69,293,920$ \\
\hline Discarded reads associated with the adapter & & & $1,043,798$ \\
\hline Clean data/raw data $(\%)$ & & & \\
\hline
\end{tabular}

$\mathrm{N}$, unknown bases more than 10\%; Q20, recognition reliability of a base is equal to $99.0 \%$; Q30, recognition reliability of a base is equal to $99.99 \%$; fq $1 / 2$, file $1 / 2$ of pair-end sequencing data; GC, the combination of bases G and C.

investigations (11). Several studies have addressed the feasibility and effectiveness of pooling-based GWAS, with considerable savings in time and cost (11-13). Additionally, whole-genome sequencing across multiple samples in a population provides an unprecedented opportunity for comprehensively characterizing the polymorphic variants in the population (14).

Although the genetic contribution to T2D is well recognized, there are now at least 19 loci containing genes, which are known to increase the risk of T2D, including PPARG, KCNJ11, KCNQ1, CDKAL1, CDKN2A-2B, CDC123-CAMK1D, MTNR1B, TCF7L2, TCF2 (HNF1B), HHEX-KIF11-IDE, JAZF1, IGF2BP2, SLC30A8, THADA, ADAMTS9, WFS1, FTO, NOTCH2 and TSPAN8 (2). To date, the current set of 66 established susceptibility loci, identified primarily through large-scale GWAS (2,8,15-22), encompasses, at most,
$10 \%$ of the familial aggregation of the disease. Of the currently established susceptibility loci, nine of the loci are contained in the 19 loci-containing genes. In the present study, the genomes of 100 Chinese patients with T2D and 100 non-diabetic Chinese individuals were examined using high throughput genome-wide re-sequencing and DNA pooling with Illumina HiSeq 2000 (Illumina, San Diego, CA, USA). The aim of the present study was to determine the rates of susceptibility genes in $\mathrm{T} 2 \mathrm{D}$ in the Chinese population.

\section{Materials and methods}

Study populations. The present study was performed between August 2012 and the end of June 2013 at the 181st Hospital of People's Liberation Army, (Guilin, China). A total of 
Table III. Alignment of quality control data.

\begin{tabular}{lr}
\hline Whole-genome statistic & Value \\
\hline Clean reads (n) & $1,367,776,414$ \\
Clean bases (bp) & $123,099,877,260$ \\
Mapped reads & $1,325,654,972$ \\
Mapped bases (bp) & $117,572,810,280$ \\
Mapping rate (\%) & 96.92 \\
Unique reads (n) & $1,271,136,561$ \\
Unique bases (bp) & 95.89 \\
Unique rate (\%) & 11.86 \\
Duplicate reads (n) & $157,244,383$ \\
Duplicate rate (\%) & 35,221 \\
Mismatch bases (bp) & 35.70 \\
Mismatch rate (\%) & 99.88 \\
Average sequencing depth & 99.38 \\
Coverage (\%) & 97.98 \\
Coverage of at least 4X (\%) & 93.02 \\
Coverage of at least 10X (\%) & \\
Coverage of at least 20X (\%) &
\end{tabular}

200 Chinese individuals were recruited, of which half were diagnosed with T2D. All participants with T2D were unrelated, and their disease was defined by World Health Organization criteria (23). The healthy individuals had a fasting plasma glucose $<5.6 \mathrm{mmol} / \mathrm{l}$, a 2 -h oral glucose tolerance test-based plasma glucose $<7.8 \mathrm{mmol} / 1$, a body mass index $<27.5 \mathrm{~kg} / \mathrm{m}^{2}$ and blood pressure $<140 / 90 \mathrm{mmHg}$, with no antihypertensive treatment. The clinical and biochemical characteristics of the 200 individuals are presented in Table I.

Ethics. The present study was approved by the Medical and Health Research Ethics Committee of the 181st Hospital of the People's Liberation Army (Guilin, China). All participants provided informed consent for the use of their biological samples for genetic investigation.

Experimental procedure. Peripheral blood samples from the 200 volunteers were collected for genomic DNA extraction. DNA preparation followed the manufacturer's protocol (Illumina). Genomic DNA was extracted and then randomly fragmented. Following electrophoresis, DNA fragments of desired length (90 bp) were gel purified using QIAquick PCR Purification kit (Qiagen GmbH, Hilden, Germany). Adapter ligation and DNA cluster preparation were performed as part of Solexa sequencing by Beijing Genomics Institute (Shenzhen, China) (24-26).

Bioinformatics analysis. The bioinformatics analysis used the sequencing data (raw data) generated from the Illumina HiSeq 2000. First, the adapter sequence in the raw data was removed, and low quality reads, which contained too many unknown bases $(\mathrm{N})$ or low quality bases were discarded. This step produced 'clean data'. Secondly, Burrows-Wheeler Aligner (BWA) (27) was used to align the reads to the reference sequence. The alignment information was stored in
Table IV. Single nucleotide polymorphism data.

\begin{tabular}{lr}
\hline Category & Value \\
\hline Total (n) & $3,974,307$ \\
1000 genome and dbSNP135 (n) & $3,911,119$ \\
1000 genome-specific (n) & 1,712 \\
dbSNP135-specific (n) & 58,466 \\
dbSNP rate (\%) & 99.88 \\
Novel (n) & 3,010 \\
Homozygous (n) & 475,874 \\
Heterozygous (n) & $3,498,433$ \\
Synonymous (n) & 11,723 \\
Missense (n) & 9,897 \\
Stopgain (n) & 76 \\
Stoploss (n) & 31 \\
Exonic (n) & 21,422 \\
Exonic and splicing (n) & 305 \\
Splicing (n) & 155 \\
ncRNA (n) & 97,213 \\
UTR5 (n) & 4,043 \\
UTR5 and UTR3 (n) & 14 \\
UTR3 (n) & 25,860 \\
Intronic (n) & $1,382,366$ \\
Upstream (n) & 18,977 \\
Upstream and downstream (n) & 2.165 \\
Downstream (n) & 2.1030 \\
Intergenic (n) & 2.1043 \\
Sorting intolerant from tolerant (n) \\
Ti/Tv (n) & 1.1923 \\
dbSNP Ti/Tv (n) & \\
Novel Ti/Tv (n) & \\
& \\
&
\end{tabular}

UTR, untranslated region; ncRNA, non-coding RNA; dbSNP, SNP database; $\mathrm{Ti} / \mathrm{Tv}$, ratio of transition to transversion.

BAM format files, which were further processed during subsequent steps, including fixing mate-pair information, adding read group information and marking duplicate reads caused by polymerase chain reaction (PCR). Following these processes, the final BAM files were ready for variant calling. Single nucleotide polymorphisms (SNPs) were detected using SOAPsnp (28), small insertion/deletions (InDels) were detected using SAMtools (29)/GATK (30,31), copy number variants (CNVs) were detected using CNVnator $(32,33)$, single nucleotide variants (SNVs) were detected using Varscan (34) and somatic InDels were detected using GATK. Structure variants (SVs) and somatic CNVs were identified using BreakDancer (35)/CREST/SeekSV (self-method) and a self-method based on the SegSeq (36) algorithm, respectively. Virus integration sites were identified using a self-method based on unmapped reads. The procedure also included purity estimation. Subsequently, filters were applied to obtain variant results of higher confidence and, based on which subsequent advanced analysis could be performed, ANNOVAR (37) was used to annotate the variant results. Quality control was required at 
Table V. List of 77 single nucleotide polymorphism loci in 37 genes identified in the present study.

\begin{tabular}{|c|c|c|c|c|c|c|c|c|c|}
\hline Gene & Function & Exonic function & dbSNP135 & SIFT & PolyPhen2 & Chr & Ref & Obs & Het/hom \\
\hline \multirow{3}{*}{ ANK1 } & Exonic & Synonymous SNV & rs 2304880 & & & 8 & G & A & Het \\
\hline & Exonic & Synonymous SNV & rs 2304873 & & & & $\mathrm{C}$ & $\mathrm{T}$ & Het \\
\hline & Exonic & Synonymous SNV & rs2304871 & & & & G & $\mathrm{A}$ & Het \\
\hline \multirow[t]{2}{*}{ ANKRD55 } & Exonic & Synonymous SNV & rs321775 & & & 5 & $\mathrm{~T}$ & $\mathrm{C}$ & Het \\
\hline & Exonic & Nonsynonymous SNV & rs 321776 & 1 & 0 & & $\mathrm{C}$ & $\mathrm{T}$ & Het \\
\hline \multirow[t]{2}{*}{ BCAR1 } & Exonic & Synonymous SNV & rs3169330 & & & 16 & A & G & Hom \\
\hline & exonic & Synonymous SNV & rs3743613 & & & & $\mathrm{C}$ & $\mathrm{T}$ & Het \\
\hline GRB14 & Exonic & Nonsynonymous SNV & rs61748245 & 0.27 & 0.009 & 2 & A & $\mathrm{T}$ & Het \\
\hline CAMK1D & Exonic & Synonymous SNV & rs 1757051 & & & 10 & $\mathrm{C}$ & $\mathrm{G}$ & Het \\
\hline \multirow[t]{4}{*}{ TSPAN8 } & Exonic & Nonsynonymous SNV & rs 1051334 & 1 & 0 & 12 & A & $\mathrm{C}$ & Het \\
\hline & Exonic & Synonymous SNV & rs2270587 & & & & $\mathrm{G}$ & A & Het \\
\hline & Exonic & Nonsynonymous SNV & rs3763978 & 0.08 & 0.981 & & $\mathrm{C}$ & $\mathrm{G}$ & Het \\
\hline & Exonic & Nonsynonymous SNV & rs79443892 & 0.73 & 0 & & $\mathrm{C}$ & $\mathrm{T}$ & Het \\
\hline \multirow[t]{3}{*}{ THADA } & Exonic & Nonsynonymous SNV & rs 17031056 & 0.34 & & 2 & $\mathrm{C}$ & $\mathrm{T}$ & Het \\
\hline & Exonic & Synonymous SNV & rs11899823 & & & & A & G & Het \\
\hline & Exonic & Synonymous SNV & rs 13021894 & & & & $\mathrm{~T}$ & $\mathrm{C}$ & Het \\
\hline \multirow[t]{2}{*}{ ADAMTS9 } & Exonic & Nonsynonymous SNV & rs 17070905 & & 0.057 & 3 & $\mathrm{C}$ & $\mathrm{T}$ & Het \\
\hline & Exonic & Nonsynonymous SNV & rs6787633 & & 0 & & G & $\mathrm{C}$ & Het \\
\hline BCL11A & Exonic & Synonymous SNV & rs7569946 & & & 2 & $\mathrm{~A}$ & $\mathrm{G}$ & Hom \\
\hline KCNQ1 & Exonic & Synonymous SNV & rs 1057128 & & & 11 & G & A & Het \\
\hline \multirow[t]{5}{*}{ HNF1A } & Exonic & Synonymous SNV & rs1169289 & & & 12 & $\mathrm{C}$ & $\mathrm{G}$ & Het \\
\hline & Exonic & Nonsynonymous SNV & rs 1169288 & 0.09 & 0.052 & & A & $\mathrm{C}$ & Het \\
\hline & Exonic & Synonymous SNV & rs2259820 & & & & $\mathrm{C}$ & $\mathrm{T}$ & Het \\
\hline & Exonic & Nonsynonymous SNV & rs2464196 & 0.06 & 0.053 & & G & $\mathrm{A}$ & Het \\
\hline & Exonic & Nonsynonymous SNV & rs 1169305 & 0.4 & 0.423999 & & A & $\mathrm{G}$ & Hom \\
\hline \multirow[t]{2}{*}{ PRC1 } & Exonic & Nonsynonymous SNV & rs 7172758 & 1 & 0 & 15 & G & $\mathrm{T}$ & Hom \\
\hline & Exonic & Synonymous SNV & rs2301826 & & & & $\mathrm{C}$ & $\mathrm{T}$ & Het \\
\hline \multirow[t]{4}{*}{ MADD } & Exonic & Synonymous SNV & rs326214 & & & 11 & G & $\mathrm{A}$ & Het \\
\hline & Exonic & Synonymous SNV & rs326217 & & & & $\mathrm{T}$ & $\mathrm{C}$ & Het \\
\hline & Exonic & Nonsynonymous SNV & rs 1051006 & 0.19 & 0 & & G & A & Het \\
\hline & Exonic & Synonymous SNV & rs 1017594 & & & & $\mathrm{~T}$ & $\mathrm{C}$ & Hom \\
\hline ADRA2A & Exonic & Synonymous SNV & rs 1800038 & & & 10 & $\mathrm{C}$ & A & Het \\
\hline GLIS3 & Exonic & Nonsynonymous SNV & rs806052 & 0.38 & 0 & 9 & A & G & Hom \\
\hline SLC2A2 & Exonic & Synonymous SNV & rs5398 & & & 3 & G & A & Het \\
\hline $\mathrm{C} 2 \mathrm{CD} 4 \mathrm{~B}$ & Exonic & Nonsynonymous SNV & rs8040712 & 0.34 & 0 & 15 & A & $\mathrm{C}$ & Het \\
\hline \multirow{5}{*}{ PTPRD } & Exonic & Synonymous SNV & rs2279776 & & & 9 & $\mathrm{C}$ & G & Het \\
\hline & Exonic & Synonymous SNV & rs 2281747 & & & & A & G & Het \\
\hline & Exonic & Nonsynonymous SNV & rs35929428 & 0.09 & 0.016 & & G & $\mathrm{A}$ & Het \\
\hline & Exonic & Synonymous SNV & rs7026388 & & & & $\mathrm{T}$ & $\mathrm{C}$ & Het \\
\hline & Exonic & Synonymous SNV & rs3763653 & & & & $\mathrm{G}$ & $\mathrm{A}$ & Het \\
\hline $\mathrm{C} 2 \mathrm{CD} 4 \mathrm{~B}$ & Exonic & Nonsynonymous SNV & rs8040712 & 0.34 & 0 & 15 & A & $\mathrm{C}$ & Het \\
\hline GRB14 & Exonic & Nonsynonymous SNV & rs61748245 & 0.27 & 0.009 & 2 & A & $\mathrm{T}$ & Het \\
\hline GLIS3 & Exonic & Nonsynonymous SNV & rs806052 & 0.38 & 0 & 9 & A & G & Hom \\
\hline PEPD & Exonic & Synonymous SNV & rs 17569 & & & 19 & $\mathrm{G}$ & A & Het \\
\hline FITM2 & Exonic & Synonymous SNV & rs6073401 & & & 20 & $\mathrm{~T}$ & $\mathrm{C}$ & Hom \\
\hline \multirow[t]{5}{*}{ KCNK16 } & Exonic & Nonsynonymous SNV & rs 11756091 & 0.03 & 0 & 6 & G & $\mathrm{T}$ & Het \\
\hline & Exonic & Synonymous SNV & rs 11753141 & & & & G & A & Het \\
\hline & Exonic & Nonsynonymous SNV & rs 1535500 & 0.12 & & & G & $\mathrm{T}$ & Het \\
\hline & Exonic & Synonymous SNV & rs 3734618 & & & & A & G & Het \\
\hline & Exonic & Synonymous SNV & rs3734619 & & & & $\mathrm{C}$ & $\mathrm{T}$ & Het \\
\hline MAEA & Exonic & Synonymous SNV & rs1128427 & 0.13 & & 4 & $\mathrm{~T}$ & $\mathrm{C}$ & Het \\
\hline PAX4 & Exonic & Nonsynonymous SNV & rs712701 & 1 & 0 & 7 & $\mathrm{~T}$ & $\mathrm{G}$ & Het \\
\hline
\end{tabular}


Table V. Continued.

\begin{tabular}{|c|c|c|c|c|c|c|c|c|c|}
\hline Gene & Function & Exonic Function & dbSNP135 & SIFT & PolyPhen2 & Chr & Ref & Obs & Het/hom \\
\hline \multirow[t]{2}{*}{ GCC1 } & Exonic & Synonymous SNV & rs3735644 & & & 7 & $\mathrm{G}$ & A & Het \\
\hline & Exonic & Synonymous SNV & rs3735642 & & & & A & $\mathrm{G}$ & Het \\
\hline \multirow[t]{3}{*}{ KCNJ11 } & Exonic & Nonsynonymous SNV & rs5215 & 0.31 & 0.002 & 11 & $\mathrm{C}$ & $\mathrm{T}$ & Het \\
\hline & Exonic & Synonymous SNV & rs5218 & & & & $\mathrm{G}$ & $\mathrm{A}$ & Het \\
\hline & Exonic & Nonsynonymous SNV & rs5219 & 0.36 & 0 & & $\mathrm{~T}$ & $\mathrm{C}$ & Het \\
\hline KCNQ1 & Exonic & Synonymous SNV & rs 1057128 & & & 11 & G & A & Het \\
\hline \multirow[t]{2}{*}{ CDKAL1 } & Exonic & Synonymous SNV & rs9350269 & & & 6 & $\mathrm{C}$ & $\mathrm{T}$ & Het \\
\hline & Exonic & Synonymous SNV & rs9465994 & & & & $\mathrm{G}$ & A & Het \\
\hline HHEX & Exonic & Synonymous SNV & rs113121942 & & & 10 & G & A & Het \\
\hline SLC30A8 & Exonic & Nonsynonymous SNV & rs 13266634 & 0.04 & 0 & 8 & $\mathrm{C}$ & $\mathrm{T}$ & Het \\
\hline \multirow[t]{5}{*}{ WFS 1} & Exonic & Nonsynonymous SNV & rs 1801212 & 1 & 0 & 4 & G & A & Hom \\
\hline & Exonic & Synonymous SNV & rs 1801206 & & & & $\mathrm{C}$ & $\mathrm{T}$ & Hom \\
\hline & Exonic & Synonymous SNV & rs 1801214 & & & & $\mathrm{C}$ & $\mathrm{T}$ & Hom \\
\hline & Exonic & Nonsynonymous SNV & rs 734312 & 0.02 & 0.99 & & $\mathrm{G}$ & A & Het \\
\hline & Exonic & Synonymous SNV & rs 1046314 & & & & $\mathrm{G}$ & A & Hom \\
\hline TCF7L2 & Exonic & Nonsynonymous SNV & rs77961654 & 0.15 & 0.996 & 10 & $\mathrm{C}$ & A & Het \\
\hline \multirow[t]{3}{*}{ THADA } & Exonic & Nonsynonymous SNV & rs 17031056 & 0.34 & & 2 & $\mathrm{C}$ & $\mathrm{T}$ & Het \\
\hline & Exonic & Synonymous SNV & rs11899823 & & & & A & G & Het \\
\hline & Exonic & Synonymous SNV & rs 13021894 & & & & $\mathrm{~T}$ & $\mathrm{C}$ & Het \\
\hline \multirow[t]{2}{*}{ ADAMTS9 } & Exonic & Nonsynonymous SNV & rs 17070905 & & 0.057 & 3 & $\mathrm{C}$ & $\mathrm{T}$ & Het \\
\hline & Exonic & Nonsynonymous SNV & rs6787633 & & 0 & & $\mathrm{G}$ & $\mathrm{C}$ & Het \\
\hline \multirow[t]{4}{*}{ TSPAN8 } & Exonic & Nonsynonymous SNV & rs 1051334 & 1 & 0 & 12 & A & $\mathrm{C}$ & Het \\
\hline & Exonic & Synonymous SNV & rs 2270587 & & & & G & A & Het \\
\hline & Exonic & Nonsynonymous SNV & rs3763978 & 0.08 & 0.981 & & $\mathrm{C}$ & G & Het \\
\hline & Exonic & Nonsynonymous SNV & rs79443892 & 0.73 & 0 & & $\mathrm{C}$ & $\mathrm{T}$ & Het \\
\hline
\end{tabular}

SNV, single nucleotide variant; Chr, chromosome.; dbSNP, SNP database; Ref, reference genotype; Het, heterozygous; Hom, homozygous; Obs, observed.

A

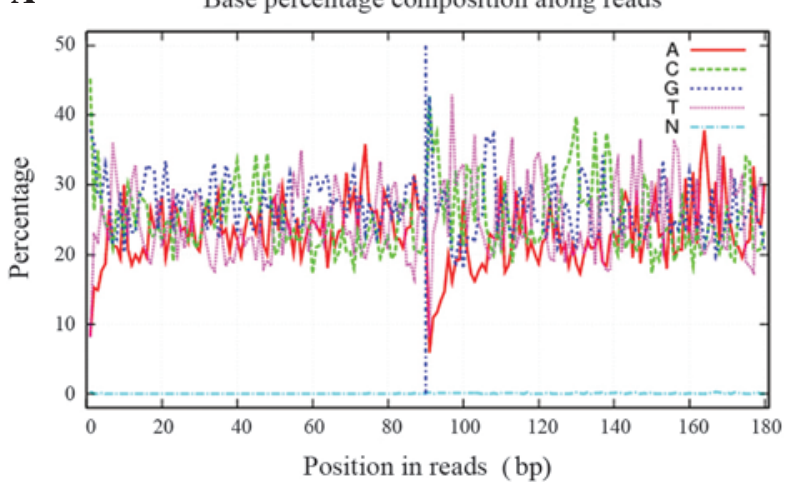

B

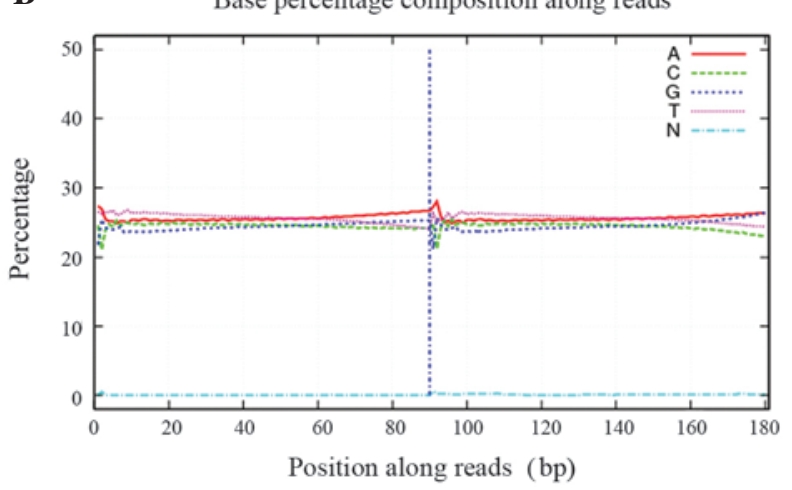

Figure 1. Base composition analysis. (A) Unbalanced base composition of raw reads. On the x-axis, position 1-90 bp represents read 1, and 91-180 bp represents read 2. Normal conditions show the A curve overlapped with the T curve, and the G curve overlapped with the C curve. Abnormal conditions during sequencing may show an unbalanced composition. (B) Balanced base composition of raw reads. On the x-axis, position 1-90 bp represents read 1, and 91-180 bp represents read 2. A balanced composition is shown, with the A curve overlapped with the $\mathrm{T}$ curve, and the $\mathrm{G}$ curve overlapped with the $\mathrm{C}$ curve.

each stage of analysis to ensure clean data, alignment and variants. SIFT (38) was used to assess the likely phenotypic effect of identified missense mutations. PolyPhen-2 (39) analysis was performed to calculate the probability of an identified mutation as deleterious for disease pathogenesis.
Sequencing quality control. The raw reads, which contained the adapter sequence, a high content of unknown bases and low quality reads were removed prior to data analysis. The filtering steps were as follows: i) Removal of adapter reads. An adapter read was defined as a read that included the adapter bases, 
Table VI. Insertion/deletion data.

\begin{tabular}{lr}
\hline Category & Value \\
\hline Total & 642,189 \\
1000 genome and dbSNP135 & 314,143 \\
1000 genome specific & 81,476 \\
dbSNP135 specific & 125,867 \\
dbSNP rate (\%) & 68.52 \\
Novel & 120,703 \\
Homozygous & 103,137 \\
Heterozygous & 539,052 \\
Frameshift insertion & 120 \\
Non-frameshift insertion & 88 \\
Frameshift deletion & 99 \\
Non-frameshift deletion & 110 \\
Frameshift block substitution & 0 \\
Non-frameshift block substitution & 0 \\
Stopgain & 2 \\
Stoploss & 1 \\
Exonic & 415 \\
Exonic and splicing & 5 \\
Splicing & 77 \\
ncRNA & 16,036 \\
UTR5 & 457 \\
UTR5 and UTR3 & 3 \\
UTR3 & 5,172 \\
Intronic & 225,732 \\
Upstream & 3,326 \\
Upstream and downstream & 102 \\
Downstream & 4,324 \\
Intergenic & 386,540 \\
\hline SNP sing & \\
\hline
\end{tabular}

SNP, single nucleotide polymorphism; UTR, untranslated region; dbSNP, dbSNP database; ncRNA, non-coding RNA.

which were removed from the raw FASTQ data; ii) removal of low-quality reads. If more than half of the bases in a read were low-quality, defined as a base quality $\leq 5$, the read was treated as a low-quality read and removed from the raw FASTQ data; iii) removal of reads in which unknown bases were $>10 \%$. The 'clean reads' were then used for downstream bioinformatics analysis. Finally statistical analysis was performed for data interpretation. The quality of the clean data is shown in Figs. 1 and 2.

Alignment quality control. The human genome build37 (hg19) was used as the reference genome in the present study. The whole-genome size of hg19 is 3,137,161,264 bp, whereas the effective size is $2,861,327,131 \mathrm{bp}$, following exclusion of the $\mathrm{N}$ bases, random regions, hap regions, and chromosome Un and chromosome $\mathrm{M}$ in the reference sequence. BWA was used to align the reference genome sequence for sequencing reads. Picard (broadinstitute.github.io/picard) was used to mark duplicated reads, which were redundant information produced by PCR.
Table VII. Structure variant data.

\begin{tabular}{lr}
\hline Category & Value \\
\hline Total & 5,590 \\
Insertion & 348 \\
Deletion & 5,002 \\
Inversion & 14 \\
ITX & 122 \\
CTX & 104 \\
Exonic & 3 \\
Exonic and splicing & 3 \\
Splicing & 7 \\
ncRNA & 133 \\
UTR5 & 3 \\
UTR5 and UTR3 & 0 \\
UTR3 & 9 \\
Intronic & 1,875 \\
Upstream & 15 \\
Upstream and downstream & 0 \\
Downstream & 31 \\
Intergenic & 3,511 \\
\hline UTR, untranslated region; ncRNA, non-coding RNA; ITX, inversion; \\
CTX, translocation. & \\
\hline
\end{tabular}

Table VIII. Copy number variant data.

\begin{tabular}{lr}
\hline Category & Value \\
\hline Total & 4,713 \\
Exonic & 930 \\
Exonic and splicing & 0 \\
Splicing & 242 \\
ncRNA & 165 \\
UTR5 & 1 \\
UTR5 and UTR3 & 0 \\
UTR3 & 9 \\
Intronic & 1,026 \\
Upstream & 56 \\
Upstream and downstream & 6 \\
Downstream & 36 \\
Intergenic & 2,242 \\
Amplification size & $13,445,200$ \\
Deletion size & $84,646,400$ \\
\hline
\end{tabular}

UTR, untranslated region, ncRNA, non-coding RNA.

\section{Results}

Quality control of sequencing data. To minimize the likelihood of systematic bias during sampling, two paired-end libraries with insert sizes of $500 \mathrm{bp}$ were prepared for all samples and were subjected to whole-genome sequencing. Each library comprised four lanes, resulting in at least 30 -fold 
A

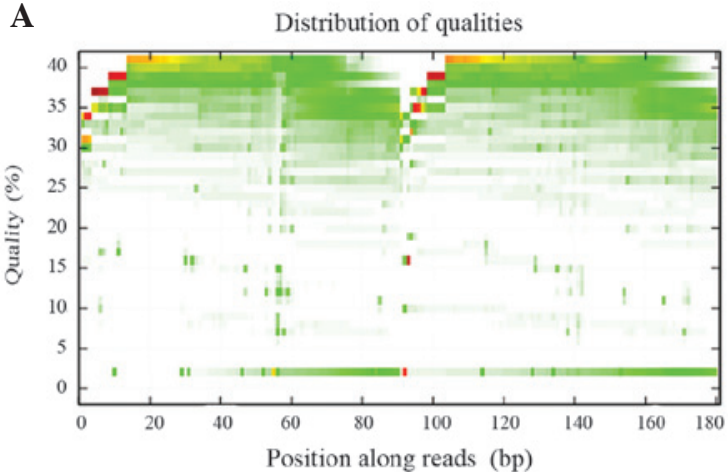

B

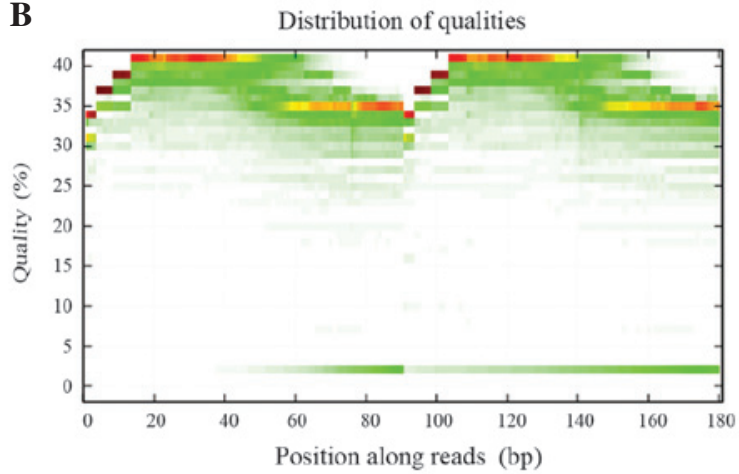

Figure 2. Evaluation of quality distribution. (A) Low quality distribution of bases along reads. On the horizontal axis, position 1-90 bp represents read 1, and 91-180 bp represents read 2. The vertical axis represents the quality value. Each dot in the image represents the quality value of the corresponding position along the reads. If the percentage of bases with low quality $(<20)$ is high, then the sequencing quality of this lane is poor. (B) If the percentage of the bases showing low quality $(<20)$ is low, then the sequencing quality of this lane is good.

Table IX. Single nucleotide variant statistics (healthy control, vs. T2D).

\begin{tabular}{lr}
\hline Category & Value \\
\hline Total & 13,049 \\
1000 genome and dbSNP135 & 12,655 \\
1000 genome specific & 11 \\
dbSNP135 specific & 282 \\
dbSNP rate (\%) & 99.14 \\
Novel & 101 \\
Hom & 0 \\
Het & 13,049 \\
Synonymous & 52 \\
Missense & 36 \\
Stopgain & 0 \\
Stoploss & 0 \\
Exonic & 88 \\
Exonic and splicing & 0 \\
Splicing & 1 \\
ncRNA & 305 \\
UTR5 & 15 \\
UTR5 and UTR3 & 0 \\
UTR3 & 112 \\
Intronic & 4,638 \\
Upstream & 73 \\
Upstream and downstream & 0 \\
Downstream & 7473 \\
Intergenic & 2.1188 \\
Sorting Intolerant from Tolerant & 2.1324 \\
Ti/Tv & 1.0612 \\
dbSNP Ti/Tv & \\
Novel Ti/Tv & 5 \\
\hline UTR, & \\
&
\end{tabular}

UTR, untranslated region; ncRNA, non-coding RNA; dbSNP, SNP database.

haploid coverage for each sample. The raw image files were processed using the Illumina pipeline for base calling;
Table X. Somatic insertion and deletion statistics (healthy control, vs. T2D).

\begin{tabular}{|c|c|}
\hline Category & Value \\
\hline 1000 genome and dbSNP135 & 1,249 \\
\hline 1000 genome specific & 688 \\
\hline dbSNP135 specific & 310 \\
\hline dbSNP rate $(\%)$ & 42.19 \\
\hline Novel & 1,448 \\
\hline Hom & 3,695 \\
\hline Het & 0 \\
\hline Frameshift insertion & 0 \\
\hline Non-frameshift insertion & 0 \\
\hline Frameshift deletion & 1 \\
\hline Non-frameshift deletion & 3 \\
\hline Frameshift block substitution & 0 \\
\hline Non-frameshift block substitution & 0 \\
\hline Stopgain & 0 \\
\hline Stoploss & 0 \\
\hline Exonic & 4 \\
\hline Exonic and splicing & 0 \\
\hline Splicing & 1 \\
\hline ncRNA & 93 \\
\hline UTR5 & 4 \\
\hline UTR5 and UTR3 & 0 \\
\hline UTR3 & 32 \\
\hline Intronic & 1,242 \\
\hline Upstream & 16 \\
\hline Upstream and downstream & 0 \\
\hline Downstream & 27 \\
\hline Intergenic & 2,276 \\
\hline
\end{tabular}

UTR, untranslated region; ncRNA, non-coding RNA; dbSNP, SNP database.

default parameters and the sequences of each individual were generated as 90-bp-paired-end reads. A total of $144.3 \mathrm{~GB}$ raw sequence data were generated in a sequencing depth of 
Table XI. Somatic copy number variant analysis (healthy control, vs, T2D).

\begin{tabular}{lr}
\hline Category & Value \\
\hline Total & 1,884 \\
Exonic & 185 \\
Exonic and splicing & 0 \\
Splicing & 21 \\
ncRNA & 41 \\
UTR5 & 0 \\
UTR5 and UTR3 & 0 \\
UTR3 & 6 \\
Intronic & 538 \\
Upstream & 14 \\
Upstream and downstream & 0 \\
Downstream & 17 \\
Intergenic & 1,062 \\
Amplification size & $1,372,716$ \\
Deletion size & $1,879,767$ \\
\hline
\end{tabular}

UTR, untranslated region; ncRNA, non-coding RNA.

Table XII. Somatic structure variant statistics (healthy control, vs. T2D).

\begin{tabular}{lr}
\hline Category & Value \\
\hline Total & 74 \\
Insertion & 6 \\
Deletion & 58 \\
Inversion & 0 \\
ITX & 2 \\
CTX & 8 \\
Exonic & 0 \\
Exonic and splicing & 0 \\
Splicing & 0 \\
ncRNA & 0 \\
UTR5 & 0 \\
UTR5 and UTR3 & 0 \\
UTR3 & 0 \\
Intronic & 24 \\
Upstream & 0 \\
Upstream and downstream & 0 \\
Downstream & 0 \\
Intergenic & 50 \\
\hline
\end{tabular}

UTR, untranslated region, ncRNA, non-coding RNA.

$\sim 30$-fold. As shown in Table II, comparison was performed between the raw and clean data, which were detected using whole-genome re-sequencing.

Alignment of quality control data. The resulting calculated T2D genome consensus sequence covered $99.88 \%$ of the hg19
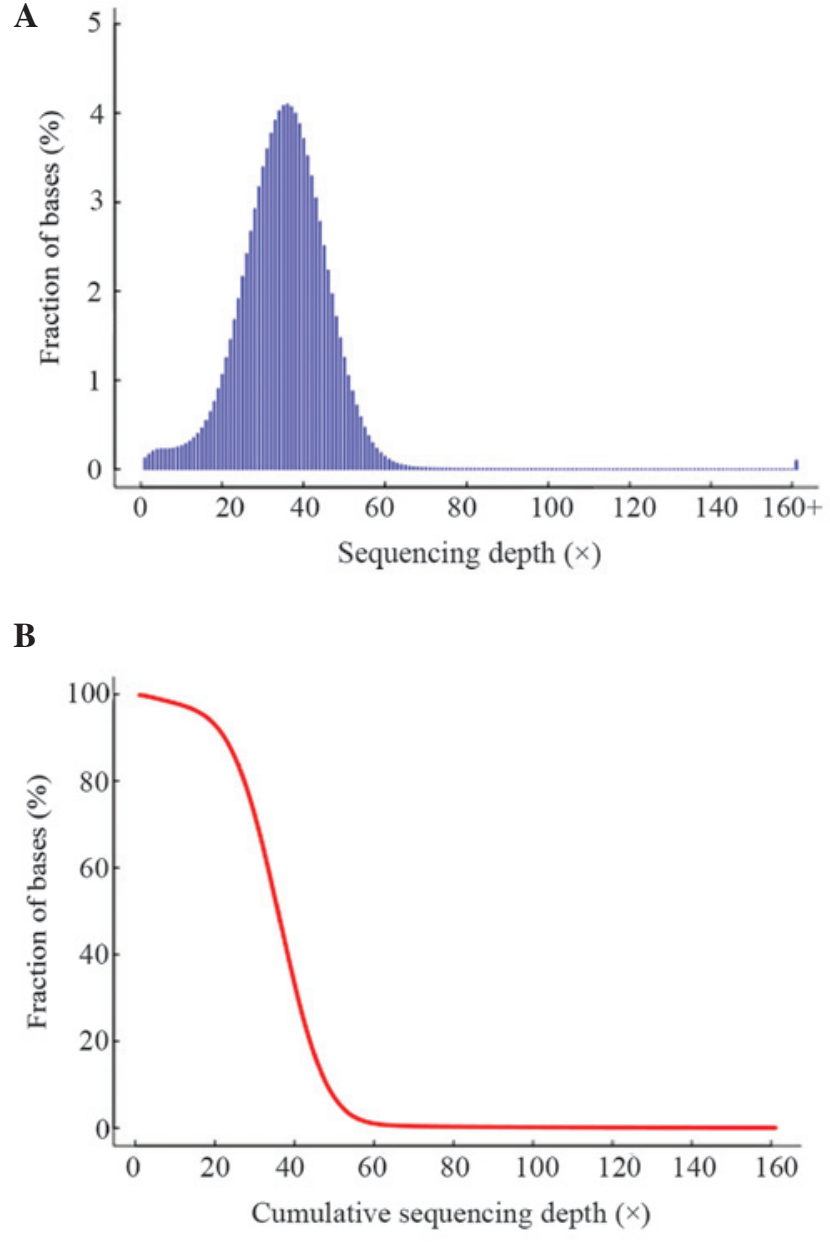

Figure 3. Distribution of per-base sequencing depth and cumulative depth distribution. (A) Distribution of per-base sequencing depth. The x-axis denotes sequencing depth, the $y$-axis indicates the percentage of the total target region at a given sequencing depth. (B) Plot of cumulative depth distribution in target regions. The $\mathrm{x}$-axis denotes sequencing depth, the $\mathrm{y}$-axis indicates the fraction of bases at or above a given sequencing depth.

sequence. At a depth of 10 -fold, the assembled consensus covered $97.98 \%$ of the reference genome using two paired-end reads. Thus, increased sequencing depth provided only a marginal increase in genome coverage. The alignment quality control results are shown in Table III. The distribution of per-base sequencing depth and cumulative depth distribution in the target regions are presented in Fig. 3. The data approximately followed a Poisson distribution, which showed that the exome-capturing target region was evenly sampled.

SNP analysis. The genotype with the highest probability at a given locus was identified for each individual sequencing sample, and the consensus sequence of the sample was assembled and saved in CNS format. Using the consensus sequence, the polymorphic loci between the identified genotype and the reference were filtered and highlighted; this constitutes the high confident SNP dataset. Following the identification of the SNPs, ANNOVAR was used to perform annotation and classification.

The results revealed that $99.88 \%$ of the T2D SNPs were present in dbSNP, and there were 3,010 novel SNPs (Table IV). A total of 485 SNPs were screened, for which the SIFT score 

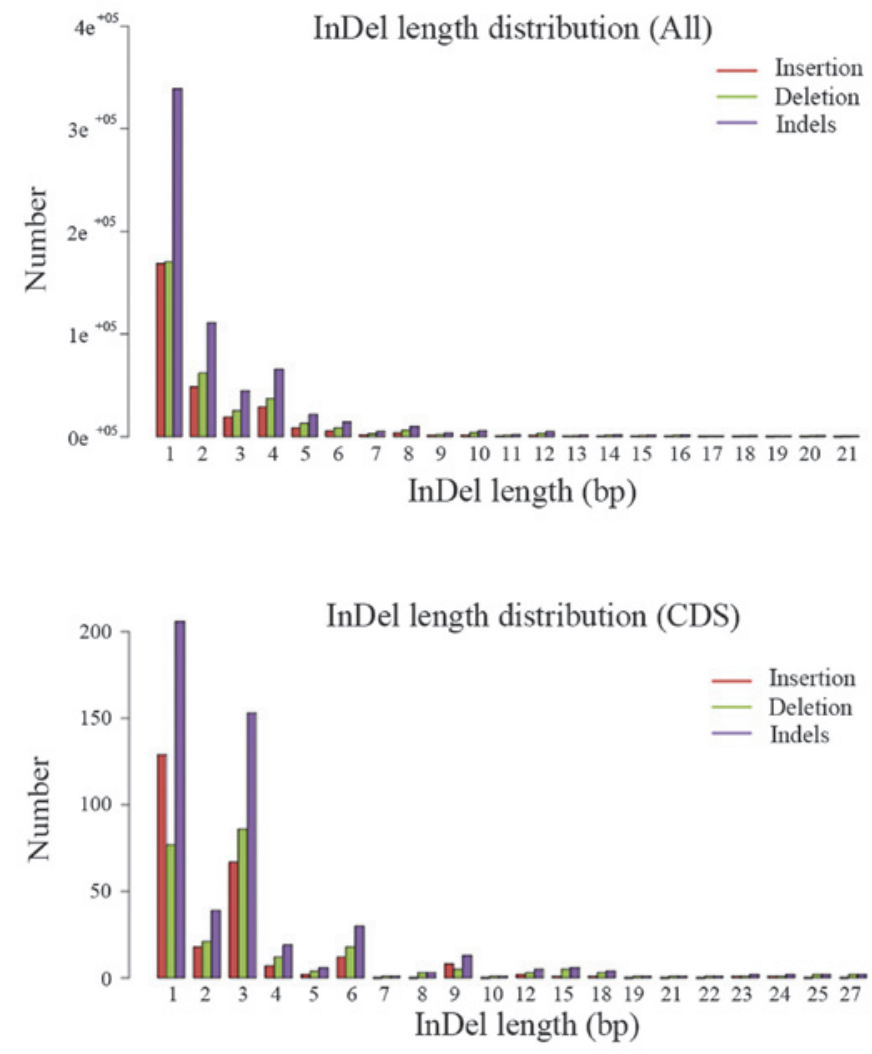

Figure 4. Length distribution of InDels in the CDS region. The results show the numbers of peaks present at specific InDel lengths. InDels with this periodicity are non-frameshift InDels, which have a relatively small effect on the genome, compared with frameshift InDels. InDel, insertion and deletion; CDS, coding sequence.

was $<0.05$, and the PolyPhen score was $>0.85$. These features suggested the pathological nature of the identified genetic variation. Of these 485 SNPs, 480 SNPs were found at exonic regions. The remaining SNPs were at exonic and splicing regions. All the SNPs were nonsynonymous genes. Compared with the 76 loci-containing genes causing an increased risk of T2D, 77 SNP loci were identified in 37 genes (Table V).

InDel analysis. To detect the InDels, the present study used pair-end reads for gap alignment using the mpileup program in SAM tools. Following identification of the InDels, ANNOVAR was used for annotation and classification. Of the 642,189 identified InDels, the percentage that overlapped the dbSNP InDels was $68.52 \%$ (Table VI). The length distribution of the InDels in the whole target region and CDS region were also plotted (Fig. 4). The length distribution of InDel in CDS region indicated that peaks are present at 3, 6 and 9 bp length, the InDels with this periodicity are non-framenshift InDels, they have relatively small effect on the genome comparing with frameshift InDels.

$S V, C N V$ and $S N V$ analyses. When aligning the paired-end reads, if a structure variation existed between the sequencing and the reference sequences, the requirements for pair-end alignment, also termed the PE map, may not be met; thus, these anomalous read pairs and soft clip reads were used in the present study to detect SVs. The resulting list of the SVs, that were detected at the whole-genome level are listed in Table VII.

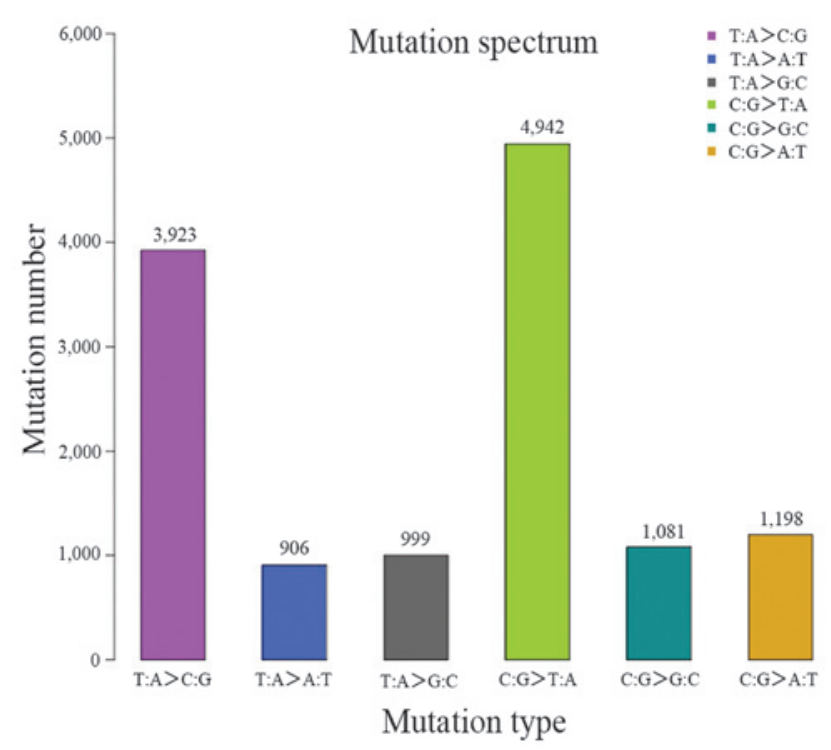

Figure 5. Somatic mutation spectrum of the whole-genome.

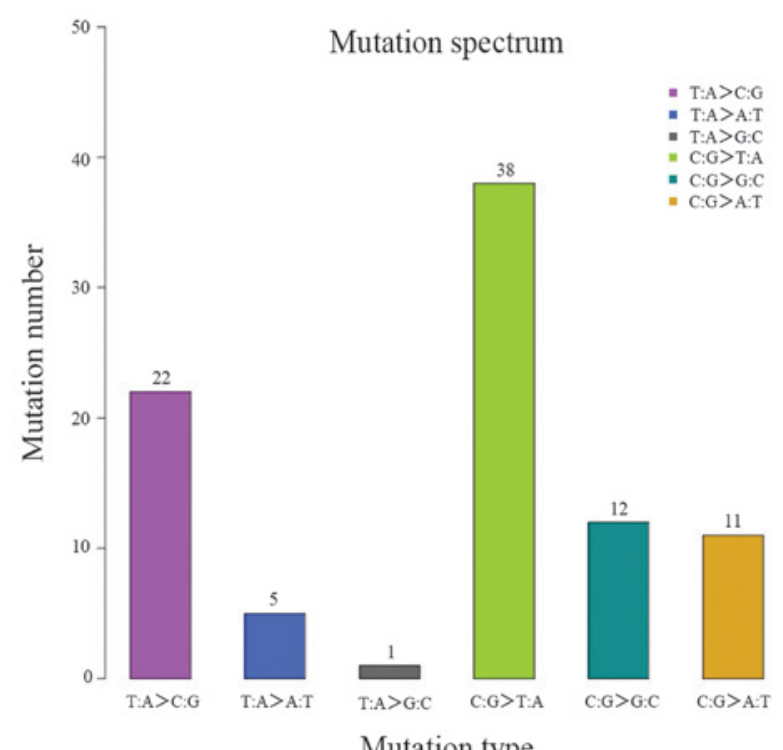

Figure 6. Somatic mutation spectrum of the CDS region.

The CNVs of each sample were detected using CNVnator. Following identification of the CNVs, ANNOVAR was used for annotation and classification (Table VIII). Varscan was used to identify specific SNVs by simultaneously comparing read counts, base quality and allele frequency between the healthy individuals and patients with type 2 diabetes. Following identification of the SNVs, ANNOVAR was again used for annotation and classification (Table IX and Figs. 5 and 6).

Analyses of somatic InDels, somatic CNVs and somatic SVs. In the sufficiently covered sites, the initial call was produced in the type 2 diabetes sample and then compared with the normal sample to detect evidence for the event. If there was no evidence to support the InDel event in the normal sample, the site was considered to be a putative somatic InDel. In total, there were 1,249 somatic InDels in 1,000 genome and 


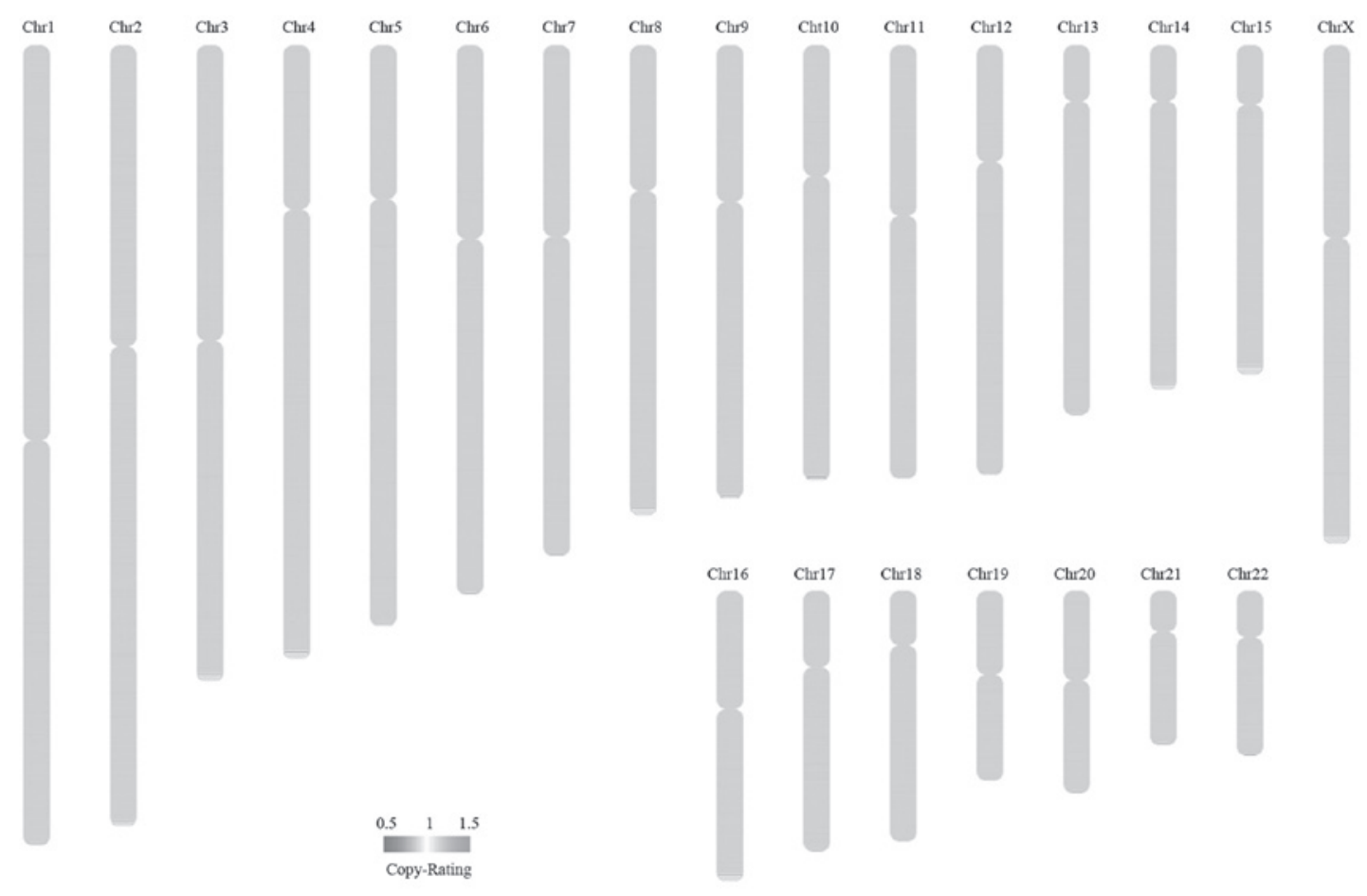

Figure 7. Overview of somatic copy number variants. Chr, chromosome.

$\mathrm{dbSNP} 135,688$ in 1,000 genome specific, and $310 \mathrm{dbSNP135}$ specific. The results of the statistical analyses are provided in Table $\mathrm{X}$. The dbSNP rate was $42.19 \%$, and without heterozygous.

Somatic CNVs correspond to relatively large regions of the genome, which are either deleted and fewer than the normal number, or duplicated and more than the normal number, on certain chromosomes. The results of the somatic CNVs analyses are shown in Table XI, and the somatic CNV overview is plotted in Fig. 7.

In the sufficiently covered sites, the initial call was produced in the type 2 diabetes sample and then compared with the normal sample to detect evidence for the event. If there was no evidence to support the SV event in the normal sample, this event was considered to be a putative somatic SV. The results of the somatic SV statistical analyses are presented in Table XII.

\section{Discussion}

In the present study, whole-genome re-sequencing was performed with DNA pooling to investigate T2D in Chinese individuals. In total, 1.44 GB of raw data were generated in a short period of time. Among the data obtained, 3,010 novel SNPs and 120,703 novel InDels were found. In addition, 5,590 SVs, 4,713 CNVs and 13,049 SNVs were identified. There was a significant difference between cases and controls in 1,884 somatic CNVs and 74 somatic SVs. These findings improve current understanding of the genetic basis of T2D and offer insight for future investigations.

Among the identified genes, only rs734312 in WFS1 (with a SIFT score 0.02 and a PolyPhen score of 0.99) suggested a pathologic nature. It was also found that, even in the same genes, the associated loci were different in the present study. Although $>30$ genetic susceptibility loci have been found in the comparison of 76 genes, the most frequently reported variants have small to moderate effects, and account for only a small proportion of the heritability of $\mathrm{T} 2 \mathrm{D}$, suggesting that the majority of inter-person genetic variation in this disease remains to be elucidated (20).

KCNQ1 (40), UBE2E2 and C2CD4A-C2CD4B (19) have been identified as T2D susceptibility genes in three GWA scans in Japanese individuals. The combined analyses identified GLIS3, PEPD, FITM2-R3HDML-HNF4A, KCNK16, MAEA, GCC1-PAX4, PSMD6 and ZFAND3 as T2D loci reaching genome-wide significance in East Asia (22). PTPRD and SRR have been identified as diabetes susceptibility loci in a study of a Han Chinese population (2). In the present study, the SNP loci in the UBE2E2, PSMD6, ZFAND3 and SRR genes were not found. The results of the present study suggested that, in different patient populations, different genes may confer risks for diabetes, which may lead to more complex study designs for investigating the molecular pathogenesis of T2D.

A simple, but important observation was that DNA pooling provides a highly effective approach for examining the genetic underpinnings of common familial diseases. DNA pooling has been confirmed to be an effective and efficient method to select candidate susceptibility loci for follow-up by individual genotyping $(12,13,41)$. This indicates that the use of GWAS for a large number of cases and controls are technically and financially feasible. Additional findings of particular interest include the large-scale examination of possible genetic variants. The present study demonstrated novel, significant associations, including SNPs, CNVs, InDels and SNVs.

The present study indicated general recommendations, which are relevant to whole-genome re-sequencing using 
DNA pooling. The first recommendation is associated with the importance of careful quality control. In the present study, 144.3 GB of raw data were generated from the Illumina pipeline, which contained too many Ns or low quality bases. Small systematic differences can readily produce effects capable of obscuring true associations from being identified $(42,43)$. The present study implemented extensive quality control checks to minimize differences in the clean data, alignment and called variants.

The sequencing method used in the present study also resulted in sequence redundancy reaching an average of 35.70-fold. Thus, the consensus sequence accuracy was higher and particularly suitable for calling heterozygous alleles. Whole-genome re-sequencing with DNA pooling technologies is high throughput technique, as one hundred million DNA fragments can be sequenced in parallel on one chip. The Illumina HiSeq 2000 platform from Illumina used in the present study can provide up to 55 GB of high-quality data per day. In this regard, it was possible to undertake comprehensive assessments of the variants within the regions of interest using this high-throughput and time efficient method.

Thirty years ago, James V. Neel (44) labeled T2D as 'the geneticist's nightmare', describing the identification of genetic factors in T2D as challenging. Numerous investigations on candidate genes for T2D have been published; however, the various approaches, including high-throughput gene scanning and gene and pedigree analysis have not been entirely successful in identifying robustly replicating T2D-susceptibility loci. Ultimately, with large samples and worldwide collaboration, novel risk factors for diabetes are likely be identified using whole-genome re-sequencing technology.

\section{Acknowledgements}

The authors would like to thank Professor Yong Dai (Department of Clinical Medical Research Center, The Second Clinical Medical College of Jinan University, Shenzhen People's Hospital, Shenzhen, Guangdong, China) and Professor Yue-ying Xiang (Department of Health Management Center, 181st Hospital, Guilin, Guangxi, China) for their helpful comments, and Dr Ming-lin Ou and Mr Xian-liang Hou from the Nephrology Department of 181st Hospital and Guangxi Key Laboratory of Metabolic Diseases Research (Guilin, China) for their technical assistance.

\section{References}

1. Chen L, Magliano DJ and Zimmet PZ: The worldwide epidemiology of type 2 diabetes mellitus-present and future perspectives. Nat Rev Endocrinol 8: 228-236, 2011.

2. Tsai FJ, Yang CF, Chen CC, Chuang LM, Lu CH, Chang CT, Wang TY, Chen RH, Shiu CF and Liu YM, et al: A genome-wide association study identifies susceptibility variants for type 2 diabetes in Han Chinese. PLoS Genet 6: e1000847, 2010.

3. Scott LJ, Mohlke KL, Bonnycastle LL, Willer CJ,Li Y,Duren WL, Erdos MR, Stringham HM, Chines PS, Jackson AU, et al: A genome-wide association study of type 2 diabetes in Finns detects multiple susceptibility variants. Science 316: 1341-1345, 2007.

4. Diabetes Genetics Initiative of Broad Institute of Harvard and MIT, Lund University, and Novartis Institutes of BioMedical Research, Saxena R, Voight BF, Lyssenko V, Burtt NP, de Bakker PI, Chen H, Roix JJ, Kathiresan S, Hirschhorn JN, et al: Genome-wide association analysis identifies loci for type 2 diabetes and triglyceride levels. Science 316: 1331-1336, 2007.
5. Sladek R, Rocheleau G, Rung J, Dina C, Shen L, Serre D, Boutin P, Vincent D, Belisle A, Hadjadj S, et al: A genome-wide association study identifies novel risk loci for type 2 diabetes. Nature 445: 881-885, 2007.

6. Zeggini E, Weedon MN, Lindgren CM, Frayling TM, Elliott KS, Lango H, Timpson NJ, Perry JR, Rayner NW, Freathy RM, et al: Replication of genome-wide association signals in UK samples reveals risk loci for type 2 diabetes. Science 316: 1336-1341, 2007.

7. Wellcome Trust Case Control Consortium: Genome-wide association study of 14,000 cases of seven common diseases and 3,000 shared controls. Nature 447: 661-678, 2007.

8. Zeggini E, Scott LJ, Saxena R, Voight BF, Marchini JL, Hu T, de Bakker PI, Abecasis GR, Almgren P, Andersen G, et al: Meta-analysis of genome-wide association data and large-scale replication identifies additional susceptibility loci for type 2 diabetes. Nat Genet 40: 638-645, 2008.

9. Gudmundsson J, Sulem P, Steinthorsdottir V, Bergthorsson JT, Thorleifsson G, Manolescu A, Rafnar T, Gudbjartsson D, Agnarsson BA, Baker A, et al: Two variants on chromosome 17 confer prostate cancer risk and the one in TCF2 protects against type 2 diabetes. Nat Genet 39: 977-983, 2007.

10. Saxena R, Elbers CC, Guo Y, Peter I, Gaunt TR, Mega JL, Lanktree MB, Tare A, Castillo BA, Li YR, et al: Large-scale gene-centric meta-analysis across 39 studies identifies type 2 diabetes loci. Am J Hum Genet 90: 410-425, 2012.

11. Baum AE, Akula N, Cabanero M, Cardona I, Corona W, Klemens B, Schulze TG, Cichon S, Rietschel M, Nöthen MM, et al: A genome-wide association study implicates diacylglycerol kinase eta (DGKH) and several other genes in the etiology of bipolar disorder. Mol Psychiatry 13: 197-207, 2008.

12. Galvan A, Falvella FS, Frullanti E, Spinola M, Incarbone M, Nosotti M, Santambrogio L, Conti B, Pastorino U, Gonzalez-Neira A and Dragani TA: Genome-wide association study in discordant sibships identifies multiple inherited susceptibility alleles linked to lung cancer. Carcinogenesis 31: 462-465, 2010.

13. Forstbauer LM, Brockschmidt FF, Moskvina V, Herold C, Redler S, Herzog A, Hillmer AM, Meesters C, Heilmann S, Albert F, et al: Genome-wide pooling approach identifies SPATA5 as a new susceptibility locus for alopecia areata. Eur J Hum Genet 20: 326-332, 2012.

14. Wong LP, Ong RT, Poh WT, Liu X, Chen P, Li R, Lam KK, Pillai NE, Sim KS, Xu H, et al: Deep whole-genome sequencing of 100 southeast asian malays. Am J Hum Genet 92: 52-66, 2013.

15. Kong A, Steinthorsdottir V, Masson G, Thorleifsson G, Sulem P, Besenbacher S, Jonasdottir A, Sigurdsson A, Kristinsson KT, Jonasdottir A, et al: Parental origin of sequence variants associated with complex diseases. Nature 462: 868-874, 2009.

16. Voight BF, Scott LJ, Steinthorsdottir V, Morris AP, Dina C, Welch RP, Zeggini E, Huth C, Aulchenko YS, Thorleifsson G, et al: Twelve type 2 diabetes susceptibility loci identified through large-scale association analysis. Nat Genet 42: $579-589,2010$.

17. Dupuis J, Langenberg C, Prokopenko I, Saxena R, Soranzo N, Jackson AU, Wheeler E, Glazer NL, Bouatia-Naji N, Gloyn AL, et al: New genetic loci implicated in fasting glucose homeostasis and their impact on type 2 diabetes risk. Nat Genet 42: 105-116, 2010.

18. Qi L, Cornelis MC, Kraft P, Stanya KJ, Linda Kao WH, Pankow JS, Dupuis J, Florez JC, Fox CS, Paré G, et al: Genetic variants at 2 q24 are associated with susceptibility to type 2 diabetes. Hum Mol Genet 19: 2706-2715, 2010.

19. Yamauchi T, Hara K, Maeda S, Yasuda K, Takahashi A, Horikoshi M, Nakamura M, Fujita H, Grarup N, Cauchi S, et al: A genome-wide association study in the Japanese population identifies susceptibility loci for type 2 diabetes at UBE2E2 and C2CD4A-C2CD4B. Nat Genet 42: 864-868, 2010.

20. Shu XO, Long J, Cai Q, Qi L, Xiang YB, Cho YS, Tai ES, Li X, Lin X, Chow WH, et al: Identification of new genetic risk variants for type 2 diabetes. PLoS Genet 6: e1001127, 2010.

21. Kooner JS, Saleheen D, Sim X, Sehmi J, Zhang W, Frossard P, Been LF, Chia KS, Dimas AS, Hassanali N, et al: Genome-wide association study in individuals of South Asian ancestry identifies six new type 2 diabetes susceptibility loci. Nat Genet 43 : 984-989, 2011.

22. Cho YS, Chen CH, Hu C, Long J, Ong RT, Sim X, Takeuchi F, Wu Y, Go MJ, Yamauchi T, et al: Meta-analysis of genome-wide association studies identifies eight new loci for type 2 diabetes in east Asians. Nat Genet 44: 67-72, 2011. 
23. Alberti KG and Zimmet PZ: Definition, diagnosis and classification of diabetes mellitus and its complications. Part 1: diagnosis and classification of diabetes mellitus provisional report of a WHO consultation. Diabet Med 15: 539-553, 1998.

24. Mardis ER: Next-generation DNA sequencing methods. Annu Rev Genomics Hum Genet 9: 387-402, 2008.

25. Wang J, Wang W, Li R, Li Y, Tian G, Goodman L, Fan W, Zhang J, Li J, Zhang J, et al: The diploid genome sequence of an Asian individual. Nature 456: 60-65, 2008.

26. Wheeler DA, Srinivasan M, Egholm M, Shen Y, Chen L, McGuire A, He W, Chen YJ, Makhijani V, Roth GT, et al: The complete genome of an individual by massively parallel DNA sequencing. Nature 452: 872-876, 2008.

27. Li H and Durbin R: Fast and accurate short read alignment with Burrows-Wheeler transform. Bioinformatics 25: 1754-1760,2009.

28. Li R, Li Y, Fang X, Yang H, Wang J, Kristiansen K and Wang J: SNP detection for massively parallel whole-genome resequencing. Genome Res 19: 1124-1132, 2009.

29. Li H, Handsaker B, Wysoker A, Fennell T, Ruan J, Homer N, Marth G, Abecasis G and Durbin R: 1000 Genome project data processing subgroup: The sequence alignment/Map format and SAMtools. Bioinformatics 25: 2078-2079, 2009.

30. McKenna A, Hanna M, Banks E, Sivachenko A, Cibulskis K, Kernytsky A, Garimella K, Altshuler D, Gabriel S, Daly M and DePristo MA: The Genome Analysis Toolkit: A MapReduce framework for analyzing next-generation DNA sequencing data. Genome Res 20: 1297-1303, 2010.

31. DePristo MA, Banks E, Poplin R, Garimella KV, Maguire JR, Hartl C, Philippakis AA, del Angel G, Rivas MA and Hanna M: A framework for variation discovery and genotyping using next-generation DNA sequencing data. Nat Genet 43: 491-498, 2011.

32. Abyzov A, Urban AE, Snyder M and Gerstein M: CNVnator: An approach to discover, genotype, and characterize typical and atypical $\mathrm{CNVs}$ from family and population genome sequencing. Genome Res 21: 974-984, 2011.

33. Mills RE, Walter K, Stewart C, Handsaker RE, Chen K, Alkan C, Abyzov A, Yoon SC, Ye K, Cheetham RK, et al: Mapping copy number variation by population-scale genome sequencing. Nature 470: 59-65, 2011.

34. Koboldt DC, Chen K, Wylie T, Larson DE, McLellan MD, Mardis ER, Weinstock GM, Wilson RK and Ding L: VarScan: Variant detection in massively parallel sequencing of individual and pooled samples. Bioinformatics 25: 2283-2285, 2009.
35. Chen K, Wallis JW, McLellan MD, Larson DE, Kalicki JM, Pohl CS, McGrath SD, Wendl MC, Zhang Q, Locke DP, et al: BreakDancer: An algorithm for high-resolution mapping of genomic structural variation. Nat Methods 6: 677-681, 2009.

36. Chiang DY, Getz G, Jaffe DB, O'Kelly MJ, Zhao X, Carter SL, Russ C,Nusbaum C, Meyerson M and Lander ES: High-resolution mapping of copy-number alterations with massively parallel sequencing. Nat Methods 6: 99-103, 2009.

37. Wang K, Li M and Hakonarson H: ANNOVAR: Functional annotation of genetic variants from high-throughput sequencing data. Nucleic Acids Res 38: e164, 2010.

38. Kumar P, Henikoff S and Ng PC: Predicting the effects of coding non-synonymous variants on protein function using the SIFT algorithm. Nat Protoc 4: 1073-1081, 2009.

39. Adzhubei IA,Schmidt S, PeshkinL, Ramensky VE, Gerasimova A, Bork P, Kondrashov AS and Sunyaev SR: A method and server for predicting damaging missense mutations. Nat Methods 7: 248-249, 2010

40. Yasuda K, Miyake K, Horikawa Y, Hara K, Osawa H, Furuta H, Hirota Y, Mori H, Jonsson A, Sato Y, et al: Variants in KCNQ1 are associated with susceptibility to type 2 diabetes mellitus. Nat Genet 40: 1092-1097, 2008.

41. Vélez JI, Chandrasekharappa SC, Henao E, Martinez AF, Harper U, Jones M, Solomon BD, Lopez L, Garcia G, Aguirre-Acevedo DC, et al: Pooling/bootstrap-based GWAS (pbGWAS) identifies new loci modifying the age of onset in PSEN1 p.Glu280Ala Alzheimer's disease. Mol Psychiatry 18: 568-575, 2013.

42. Clayton DG, Walker NM, Smyth DJ, Pask R, Cooper JD, Maier LM, Smink LJ, Lam AC, Ovington NR, Stevens HE, et al: Population structure, differential bias and genomic control in a large-scale, case-control association study. Nat Genet 37: 1243-1246, 2005.

43. Zondervan KT and Cardon LR: The complex interplay among factors that influence allelic association. Nat Rev Genet 5 89-100, 2004.

44. Neel JV. In: The Genetics of Diabetes Mellitus. Creutzfeldt W, Köbberling J, Neel JV, editors. Springer; Berlin: 1976. pp. 1-11. 